\title{
AUTHOR INDEX (VOLUMES 1 \& 2)
}

A

Agishtein, M.E. \& Migdal, A.A Recursive sampling of planar graphs and fractal properties of a two-dimensional quantum gravity

Aldea, N., Indrea, E. \& Borodi, G. Application of the Fourier transform in the EXAFS spec troscopy

Anderson, D.V. see Schwenn, U. Andreichenko, V.B., Dotsenko, Vl.S., Shchur, L.N. \& Talapov, A.L. Special-purpose Ising model random lattice processor

Antonov, V.N. see Krasovskii, E.E.

Aoki, S., Shrock, R., Berg, B. Bernard, C., Ogilvie, M., Petcher, D., Bhanot, G., Rossi, P., Bitar, K., Edwards, R., Heller, U.M., Kennedy, A., Sanielevici, S., Brower, R., Potvin, J., Rebbi, C., Brown, F.R., Christ, N., Mawhinnery, R., DeTar, C., Draper, T., Liu, K.-F., Gottlieb, S., Hamber, H., Kilcup, G., Shigemitsu, J., Kogut, J., Kronfeld, A., Lee, I.-H., Negele, J., Ohta, S., Sexton, J.C., Shuryak, E., Sinclair, D.K., Soni, A. \& Wilcox., W., Physics goals of the $Q C D$ Teraflop project

Aragaō de Carvalho, C. see do Amaral, M.G.

Arenzon, J.J. see Penna, T.J.P. Arribas, E., Gallardo, C., Molina, M. \& Sanjosé, V. Electric field lines

$1(1990) 165$

2 (1991) 211

2 (1991) 143

2 (1991) 805

2 (1991) 400

$2(1991) 829$

2 (1991) 561

2 (1991) 711

$2(1991) 216$

Baillie, C. Lattice spin models and new algorithms $-A$ re view of Monte Carlo computer simulations

Balashova, O. Yu see Zelenskaya, N.S.

Baranovskii, S.D., Levin, E.I.

Ruzin, I.M., Fritzsche, H. \& Shklovskii, B.1. Computer simulation of energy relation and recombination of nonequilibrium electrons in a dis ordered system with localized electronic states
Bardan, V. On the processing of non-rectangularly sampled multi-dimensional geophysical data

Barnes, T. The 2D Heisenberg antiferromagnet in high- $T_{\mathrm{c}}$ superconductivity

Barszczak, T. \& Kutner, R. Influence of the back-jump correlations on the Fermi-Dirac statistics

Baum, J.O. see CruzeiroHansson, $L$

Bekenstein, J.D. \& Schiffer, M Quantum limitations on the storage and transmission of information

Belenkii, A. Ya. \& Fradkin, M.A. Local electronic structure and short-range order in a computer simulated amorphous metal

Belyaeva, T.L. \& Zelenskaya, N.S. Computation of nuclear reactions with light-heavy ions

Berdnicov, A.S. Modified Lanczos method for optimal polynomial approximation of the solution of differential equations with polynomial coefficients

Berdnicov, A.S. \& Turtia, S.B Monte-Carlo simulation arte facts in physical modeling due to pseudo-random generators

Beresnev, I. Numerical model of the spherical elastic wave propagation in a nonlinear medium

Berg, B. see Aoki, S.

Bernard, C. see Aoki, S.

Bernicov, A.S. see Vinogradova, $S . A$.

Berruyer, G. \& Hammersley, A. Parallelism of an interactive fitting algorithm

Besold, G. see Schleier, W.

Bhanot, G. see Aoki, S.

Bhanot, G.V., Parikh, J.C., Sheorey, V.B. \& Pandey, A Quantum chaos and sensitivity to system parameters

Bien, R., Fuchs, B. \& Wielen, R. High spatial resolution us ing the conventional particlemesh technique

2 (1991) 223

$2(1991) 659$

2 (1991) 227

2 (1991) 305

$1(1990) 355$

$2(1991) 232$

$2(1991) 238$

$2(1991) 243$

2 (1991) 246

2 (1991) 250

2 (1991) 829

2 (1991) 829

$2(1991) 527$

2 (1991) 254

2 (1991) 468

2 (1991) 829

$1(1990) 279$

2 (1991) 3

Binder, $K$ Simulation of dense polymer systems in two and three dimensions

2 (1991) 263 
Bishop, N.T. The characteristic initial value problem of general relativity as a benchmark for MIMD machines

Bitar, K. see Aoki, S.

Blöte, H.W.J. Statistical mechanics and special-purpose computers

Blöte, H.W.J. see Smit, T.S

Bokelmann, G.H.R. \& Silver, P.G. A procedure for the extraction of multiple body wave traveltimes from seismological waveform data

Bokhove, O. see Compagner, A.

Bonomi, E., Fluck, M., Gruber,

R., Herbin, R., Richner, T., Schmid, V. \& Tran, C.T. ASTRID: A programming environment for physicists

Boris, J.P. see Oran, E.S

Borodi, G. see Aldea, $N$.

Bourbonnais, R. \& Maynard, R.

Anharmonic vibrations in

ordered and disordered oneand two-dimensional systems

Bourbonnais, R., Herrmann, H.J. \& Vicsek, T. Simulation of kinetic roughening with power-law noise on the connection machine

Braszczak, T. see Przenioslo, $R$.

Brindle, D. \& Care, C.M. Monte Carlo simulation of lattice model of amphiphile and solvent mixture on a transputer array

Broad, J.T. see Jetzke, $S$.

Brocks, G., Kelley P.J. \& Car, R. Ab-initio calculation of migration energies of adatoms on the Si(100) surface

Brower, R. see Aoki, $S$

Brown, F.R. see Aoki, S.

Bruin, C. see Compagner, A.

Burm, H. see Geertsema, $G$.

C

Cap, F. Collocation method to solve analytically nonseparable boundary problems

Caprasse, H., Demaret, J., Gatermann, K. \& Melenk, H Power-law type solutions of fourth-order gravity for multidimensional Bianchi I universes

Car, R. see Brocks, $G$

Care, C.M. see Brindle, D.

Cavaleru, A.O.R. see Niculescu, V.I.R.
2 (1991) 267

2 (1991) 829

2 (1991) 14

2 (1991) 487

2 (1991) 276

2 (1991) 300

2 (1991) 21

2 (1991) 430

2 (1991) 211

$1(1990) 233$

2 (1991) 719

2 (1991) 450

2 (1991) 284

2 (1991) 377

2 (1991) 288

2 (1991) 829

2 (1991) 829

2 (1991) 300

2 (1991) 353

2 (1991) 292

2 (1991) 601

2 (1991) 288

2 (1991) 284

$2(1991) 423$
Christ, N., see Aoki, $S$.

Coats, R.S. see Seidel, D.B.

Compagner, A. Random-number generation

Compagner, A. see Smit, T.S.

Compagner, A., Bruin, C. \& Bokhove, O. Molecular dynamics of unstable systems

Cook, G.O., Jr. ALPAL, a program to generate physics simulation codes from natural descriptions

Cooper, W.A. see Schwenn, $U$.

Cruzeiro-Hansson, L., Baum, J.O. \& Finney, J.L. Electron states in static disordered systems and fluid systems

2 (1991) 829

2 (1991) 475

2 (1991) 296

2 (1991) 487

2 (1991) 300

1 (1990) 1

2 (1991) 143

2 (1991) 305

Dammers, A.J. \& Radelaar, S. Simulation of polycrystalline film growth

Davydov, D.A. see Vinogrado$v a, S . A$.

de Almeida, R.M.C. see Penna T.J.P

Decker, K.M., Jayewardena, C. \& Rehmann, R. Libraries and development environments for Monte Carlo simulations of lattice gauge theories on parallel computers

Degtyarev, L.M. \& Drozdev, V.V. Adaptative mesh com putation of magnetic hydrodynamic equilibrium

Dekeyser, R. New algorithm for potentials on resistor networks and large application to dielectric breakdown

de Korvin, A. see Humpert, B

Demaret, J. see Caprasse, $H$.

Dement'ev, A.I. see Zaitsevskii, A. $V$.

Dent, D. see Simons, A.J.

de Oliveira, P.M.C. see Penna, T.J.P

DeTar, C. see Aoki, C.

Ding, H.-Q. Heavy quark potential in lattice $Q C D:$ A review of recent progress at CALTECH

do Amaral, M.G., Kischinhevsky, M., Argaō de Carvalho, C. \& Teixeira, F.L. An efficient method to calculate field theories with dynamical fermions

Dotsenko, Vl.S

see Andreichenko, V.B. 2 (1991) 805

Draper, T. see Aoki, $S . \quad 2$ (1991) 829
2 (1991) 310

2 (1991) 527

2 (1991) 711

2 (1991) 322

2 (1991) 86

2 (1991) 601

2 (1991) 546

2 (1991) 549

2 (1991) 711

2 (1991) 829

2 (1991) 637 
Drevermann, H. \& Grab, C. Graphical concepts for the representation of events in high energy physics

Drevermann, H., Grab, C., Nilsson, B.S. \& Vogl, R.K. Graphic concepts for the represention of events in high energy physics

Drozdev, V.V. see Degtyarev, L.M.

Drye, T.J. \& Tucker, J.W. Ndimensional numerical integration over a delta function

Duda, P., Grabski, J., Kotur, J., Peryt, W., Proboszcz, I. \& Zyla, Z. Computer aided physical laboratory in CAMAC system

Dumont, J.-J. \& Tomassini, M. Portability and reusability, standardized programming for present and future computers

Dunweg, B. \& Paul, W. Brownian dynamics simulations without Gaussian random numbers

E

Eastwood, J.W. 'Virtual particle' elecromagnetic particle-mesh algorithms for relativistic microwave source modelling

Edvardsson, S., Wolf, M. \& Thomas, J. Molecular dynamics simulation applied to the study of optical absortion spectra of $\mathrm{Nd}_{2} \mathrm{O}_{3}$

Edwards, R. see Aoki, $S$.

Eržen, V. see Stepisnik, J.

Etienne, F. The impact of modern graphics tool on science, and their limitations

Farberovich, O.V. see Katsnelson, A.A.

Fee, G.J. see Scott, T.C.

Finney, J.L. see CruzeiroHansson, $L$.

Flück, M see Bonomi, $E$

Fonk, Y.T.J.C. see Smit, T.S.

Fradkin, M.A. see Belenkii, A. Ya.

Frenkel, D. Fast algorithms for slow processes in lattice-gas cellular automata

Fritzsche, H. see Baranovskii, S.D.

Fu, G.Y. see Schwenn, $U$.

Fuchs, B. see Bien, $R$.
$2(1991) 817$

2 (1991) 343

2 (1991) 328

2 (1991) 30

$2(1991) 331$

2 (1991) 337

2 (1991) 39

2 (1991) 348

2 (1991) 829

2 (1991) 502

$2(1991) 58$

2 (1991) 391 $1(1990) 53$

2 (1991) 305

2 (1991) 21

2 (1991) 487

2 (1991) 232

2 (1991) 66

2 (1991) 220

2 (1991) 143

2 (1991) 3
G

Gallardo, C. see Arribas, $E$.

Gallas, J.A.C. \& Herrmann, H.J. Investigating an automaton of "class 4"

Gatermann, K. see Caprasse, H. 2 (1991) 601

Geddes, K.O. see Scott, T.C.

Geertsema, G. \& Burm, H. Porting a 3D-turbulence simulation to a multi-processor system

Gerling, R.W. \& Stauffer, D.

High speed simulations of ordered and disordered cellular automata

Gislén, L. Educational statistical physics on the computer

Glance, N., Hogg, T. \& Huberman, B.A. Computational ecosystems in a changing environment

Goedbloed, J.P. see Huysmans, $G . T . A$.

Golubok, A.O. see Vinogradova, $S$. A.

Gottlieb, S. see Aoki, S.

$2(1991) 601$
$1(1990) 53$

2 (1991) 353

2 (1991) 799

2 (1991) 361

2 (1991) 735

2 (1991) 371

2 (1991) 527

2 (1991) 829

1 (1990) 147 ;

2 (1991) 328

2 (1991) 337

Grabski, J. see Duda, $P$

Grigorenko, A.A. see Katsnelson, A.A.

Gruber, R. see Bonomi, $E$.

2 (1991) 391

2 (1991) 21

Gruber, R. see Schwenn, $U$.

Gustafsson, B. Analysis and methods in fluid mechanics

2 (1991) 143

2 (1991) 75

2 (1991) 450

H

Hamber, H. see Aoki, S.

2 (1991) 829

Hammersley, A. see Berruyer, $G$.

Hammersley, A. Parallelism of a 2-D fast Fourier transform algorithm

Hansen, J.E. see Landtman, $\boldsymbol{M}$.

Heemskerk, M.H.M. \& Savonije,

G.J. Non-axisymmetric unstable modes in a thin differential ly rotating gaseous disk

Heermann, D.W.The size of a random surface

Heinz, K. see Schleier, $W$

Heller, U.M. see Aoki, S.

Herbin, R. see Bonomi, $E$.

Heringa, J.R. see Smit, T.S.

Herrmann, H.J. see Gallas, J.A.C.

Herrmann, H.J. see Normand, $J . M$.

Herrmann, H.J. see Bourbonnais, $R$.

2 (1991) 254

2 (1991) 363

2 (1991) 403

2 (1991) 367

2 (1991) 613

2 (1991) 468

2 (1991) 829

2 (1991) 21

2 (1991) 487

$1(1990) 181$

1 (1990) 207

2 (1991) 719

Hogg, T. see Glance, $N$. 2 (1991) 735

Hoogland, A. see Smit, T.S. $\quad 2$ (1991) 487 
Hua, L. \& Pawley, G.S. A molecular dynamics study of $S F_{6}$

Huberman, B.A. see Glance, $N$.

Humpert, B. \& de Korvin, A. Optimization and expert system with neural networks

Huysmans, G.T.A., Goedbloed, J.P. \& Kerner, W. Isoparametric bicubic hermite elements for solution of the GradShafranov equation

I

Iglesias, J.R. see Penna, T.J.P. $\quad 2$ (1991) 711

Indrea, E. see Aldea, $N$

Ionescu, L. Gh. see Podoleanu, A. Gh

\section{$\mathbf{J}$}

Jayewardena, C. see Decker, K.M.

Jetzke, S. \& Broad, J.T. Evaluation of higher order matrix ele ments in atomic physics: Using convergence acceleration methods

Johnson, W.A. see Seidel, D.B.

$\mathbf{K}$

Kafka, J. \& Nguyen, V.N. Finite difference, finite element and boundary element formulae as a language of applied physics

Karule, E. Multiphoton ionization

Katsnelson, A.A. see Stepanyuk, V.S.

Katsnelson, A.A. see Trushin, O.S.

Katsnelson, A.A., Stepanyuk, V.S., Grigorenko, A.A., Farberovich, O.V. \& Szasz, A. Self-consistent calculation of electronic structure and optical properties of $A^{\prime \prime} B^{V I}$ compounds

Kelly, P.J. see Brocks, $G$.

Kennedy, A. see Aoki, $S$

Kerner, W. see Huysmans, G.T.A

Kiefer, M.L. see Seidel, D.B.

Kilcup, G. see Aoki, S.

Kischinhevsky, M. see do Amaral, M.G.

Kochan, T.A. see Soltys, J.

Kogut, J. see Aoki, S

Kohring, G.A. Large scale neural network simulations

Kohring, G.A. Parallelization of short- and long-range cellular automata on scalar, vector. SIMD and MIMD machines
$2(1991) 371$

2 (1991) 437

2 (1991) 316

2 (1991) 377

$2(1991) 475$

2 (1991) 383

1991) 413

2 (1991) 86

2 (1991) 497

$2(1991) 523$

2 (1991) 391

2 (1991) 288

2 (1991) 829

2 (1991) 371

2 (1991) 475

2 (1991) 829

2 (1991) 561

2 (1991) 491

2 (1991) 829

$1(1990) 259$

2 (1991) 755
2 (1991) 387
Koren, B. A computational tool for analyzing strong viscousinviscid interactions in gasdynamics

Kos, M. see Stepisnik, J.

2 (1991) 395

2 (1991) 502

2 (1991) 337

2 (1991) 497

Kozlov, A.V. see Stepanyuk, V.S.

Krasovskii, E.E. \& Antonov, V.N. Calculated optical properties of the anisotropic transition metals

2 (1991) 400

2 (1991) 829

Kronfeld, A. see Aoki, S

Kurdyumov, S.P. Evolution and self-organization laws in complex systems

Kutner, R. see Barszczak, $T$.

Kutner, R. see Przenioslo, $R$.

1 (1990) 299

2 (1991) 227

2 (1991) 450

Laaksonen, L. see Pyykkö, P. 2 (1991) 455

Labahn, G. see Scott, T.C.

Landtman, M., Shen, Y.T. \&

Hansen, J.E. Ab initio calculations of orthogonal parameters for complex configurations using B-splines

Lax, M. see Yudanin, $B$

Lee, I.-H see Aoki, $S$.

Lejeune, A. \& Perdang, J. A CA model for local galactic evolution

Levin, E.I. see Baranovskii, S.D.

Liu, K.-F see Aoki, $S$

$1(1990) 53$

$\mathbf{M}$

Mähönen, P. \& Punkka, V. Gravitation field calculations on a dynamic lattice by distributed computing

Manner, R. Can quantum synchronizers solve the metastability problem of asynchronous digital systems?

Marangi, C., Nardulli, G. \& Pasquariello, G. Dynamical properties of neural networks with correlated patterns

Marletta, M. see Pryce, J.D.

Martin, R.M. Derivation of models for the interacting electrons in $\mathrm{Cu}-\mathrm{O}$ superconductors

Mawhinney, R. see Aoki, S.

Maynard, R. see Bourbonnais, $R$

Meirovitch, H. The scanning simulation method for macromolecules

Melenk, H. see Caprasse, $H$.

Merazzi, S. see Schwenn, $U$.

Metcalf, M. Fortran $90-A$ summary
$2(1991) 418$

$1(1990) 329$

2 (1991) 773

2 (1991) 443

2 (1991) 105

2 (1991) 829

1 (1990) 233

$1(1990) 119$

2 (1991) 601

2 (1991) 143

$1(1990) 193$ 
Migdal, A.A. see Agistein, M.E.
Molina, M. see Arribas, E.
Monagan, M.B. see Scott, T.C.
Moore, R.A. see Scott, T.C.
Moscick, J.K. see Wnek, M.
Mountain, R.D. \& Thirumalal, D.
Ergodic convergence in liquids
and glasses

$\mathbf{N}$

Nardulli, G. see Marangi, $C$. Nayak, P. see Trushin, O.S.

Negele, J. see Aoki, $S$.

Neschen, M. An efficient multispin coding algorithm for neural networks

Nguyen, V.N. see Kafka, J.

Niculescu, V.I.R. \& Cavaleru, A.O.R. Internal location of a point in a nonconvex polygon in $3 D$

Nieminen, R.M. see von Boehm, $J$.

Nilsson, B.S. see Drevermann, $H$.

Normand, J.M. \& Hermann, H.J. Precise numerical determination of the superconducting exponent of percolation in three dimensions

\section{$\mathbf{0}$}

Ogilvie, M. see Aoki, S.

Ohta, S. see Aoki, S.

Olko, M. \& Turski, L.A. Cartesian coordinate solver for block-like equations: application to dynamics of a ID magnetic chain

Olsen, J. see Pyykkö, P.

Oran, E.S. \& Boris, J.P. Compressible flow simulation on a massively parallel computer

Ovchinnikova, A.A. see Zelenskaya, N.S.

2 (1991) 773

2 (1991) 523

2 (1991) 829

2 (1991) 623

$2(1991) 383$

2 (1991) 423

2 (1991) 272

2 (1991) 328

$1(1990) 207$

2 (1991) 829

2 (1991) 829

2 (1991) 425

2 (1991) 430

2 (1991) 552

$\mathbf{P}$

Pandey, A. see Bhanot, G.V. Parikh, J.C. see Bhanot, G.V.

$1(1990) 279$

Pasquariello, G. see Marangi, C. 2 (1991) 773

Paul, W. see Dunweg, $B$.

Pawley, G.S. see Hua, $L$.

Pawley, G.S. see Trew, A.S

Penna, T.J.P., de Oliveira,

P.M.C., Arenzon, J.J., de

Almeida, R.M.C. \& Iglesias,

J.R. Convergence time on the $R S$ model for neural networks

Perdang, J. see Lejeune, A.

Peryt, W. see Duda, P.

2 (1991) 413

2 (1991) 515

2 (1991) 711

2 (1991) 337
2 (1991) 455

Petcher, D. see Aoki, S.

Planinšic, G see Stepisnik, $J$.

Podoleanu, A. Gh. \& Ionescu, L.

Gh. A cumulative subinterval photon counting probability method for statistical characterization of light

Pogačnik, P. see Stepisnik, J.

Pointon, T.D. see Seidel, D.B.

Polyakov, V.I. see Topol, I.A.

Potvin, J. see Aoki, S.

Proboszcz, L. see Duda, P.

Pryce, J.D. \& Marletta, M. A new software package for Sturm-Liouville and Schrödinger problems

Przenioslo, R., Braszczakk, T., Kutner, R., Guzicki W. \& Renz, W. Monte Carlo simulations of lattice gases exhibiting quantum statistical distributions

2 (1991) 829

2 (1991) 502

2 (1991) 437

2 (1991) 502

2 (1991) 475

2 (1991) 510

2 (1991) 829

2 (1991) 337

2 (1991) 443

Punkka, V. see Mähönen, $P$

Putzer, A. Software engineering, data modeling and databases in physics experiments

Pyykkö, P., Sundholm, D. Laaksonen, L. \& Olsen, J. Two fully numerical methods in quantum chemistry

2 (1991) 455

\section{$\mathbf{Q}$}

Quintenz, J.P. see Seidel, D.B.

2 (1991) 475

$\mathbf{R}$

Radelaar, S. see Dammers, A.J.

Raraport, D.C. Multi-million particle molecular dynamics: design considerations for vector and distributed processing

Rebbi, C. see Aoki, S.

Reger, J.D. Quantum Monte Carlo simulations of models related to high-T $T_{\mathrm{c}}$ superconductivity on a transputer network

Rehmann, R. see Decker, K.M.

Renz, W. see Przenioslo, $R$.

Richner, T. see Bonomi, $E$.

Rossi, P. see Aoki, S.

Ruffert, M. Large viewing threedimensional hydrodynamical data by ray tracing

Ruzin, I.M. see Baranovskii, S.D.

2 (1991) 310

2 (1991) 458

2 (1991) 829

2 (1991) 132

2 (1991) 316

2 (1991) 450

2 (1991) 21

2 (1991) 829

2 (1991) 461

2 (1991) 200

$\mathbf{S}$
Sanielevici, S. see Aoki, S. 2 (1991) 829 Sanjosé, V. see Arribas, $E . \quad 2$ (1991) 216 
Savonije, G.J. see Heemskerk, M.H.M

Schiffer, M. see Bekenstein, J.D. Schleier, W., Besold, G. \&

Heinz, K. Parallel Monte Carlo algoritms for domain growth kinetics

Schmid, V. see Bonomi, E.

Schwenn, U., Cooper, W.A., Fu, G.Y., Gruber, R., Merazzi, S. \& Anderson, D.V Three-dimensional ideal magnetohydrodynamic stability on parallel machines

Scott, T.C., Moore, R.A., Fee, G.J., Monagan, M.B. Labahn, G. \& Geddes, K.O. Perturbative solutions of quantum mechanical problems by symbolic computation: A review

Seidel, D.B., Kiefer, M.L., Coats, R.S., Pointon, T.D., Quintenz, J.P. \& Johnson, W.A. The 3-D, electromagnetic, particle-in-cell code, QUICKSILVER

Sexton, J.C. see Aoki, S.

Shchur, L.N. see Andreichenko, $V . B$.

Shen, Y.T. see Landtman, $M$

Sheorey, V.B. see Bhanot, G.V.

Shigemitsu, J. see Aoki, S.

Shrock, R. see Aoki, S.

Shuryak, E. see Aoki, S.

Silver, P.G. see Bokelmann, G.H.R.

Simons, A.J., \& Dent, D. The ECMWF multi-tasking weather prediction model

Sinclair, D.K. see Aoki, $S$.

Sklovskii, B.I. see Baranovskii, $S . D$.

Slagter, R.J. Evolution of gauge strings on cylindricalsymmetric backgrounds

Smit, T.S., Heringa, J.R., Blöte, H.W.J., Compagner, A., Fonk, Y.T.J.C. \& Hoogland,

A. Monte Carlo renormalization of the 3-D-Ising model and the analyticity of block-spin transformations

Soltys, J. \& Kochan, T.A. Relaxation time analysis of a simulated binary alloy

Sommer, R. Hadron structure from lattice quantum chromo dynamics $(O C D)$

Soni, A. see Aoki, $S$.

2 (1991) 367

1 (1990) 355

2 (1991) 468

2 (1991) 21

$2(1991) 143$

$1(1990) 53$

2 (1991) 475

2 (1991) 829

2 (1991) 805

2 (1991) 403

1 (1990) 279

2 (1991) 829

2 (1991) 829

2 (1991) 829

2 (1991) 276

2 (1991) 158

2 (1991) 829

$2(1991) 220$

2 (1991) 483

2 (1991) 487

2 (1991) 491

2 (1991) 187

2 (1991) 829

2 (1991) 799

Stauffer, D. see Gerling, R.W. Stepanyuk, V.S. see Katsnelson, A.A.

2 (1991) 391
Stepanyuk, V.S. see Trushin, O.S.

Stepanyuk, V.S., Kozlov, A.V. Katsnelson, A.A \& Szasz, A. Application of a computational method in solid state physics for investigation of the electronic structure of defects in metals

2 (1991) 497

Stepišnik, J., Kos, M., Planinšix, G., Erzen, V. \& Pogačnik, P. Personal computer supervising an NMR imaging experiment without magnet

Sugar, R. Numerical studies of many electron systems

Sundholm, D. see Pyykkö, P.

Sundius, T. Molecular force field calculations on large computers

Swendsen, R.H. Acceleration algorithms in Monte Carlo simulations in statistical physics

Szasz, A. see Katsnelson, A.A.

Szasz, A. see Stepanyuk, V.S.

2 (1991) 201

2 (1991) 391

2 (1991) 497

$\mathbf{T}$

Talapov, A.L. see Andreichenko, V.B.

Teixeira, F.L. see do Amaral, M.G.

Thielemans, K. A Mathemati$\mathrm{ca}^{T M}$ package for computing operator product expansions

Thirumalal, D. see Mountain, $R D$

Thomas, J. see Edvardsson, $S$,

Timofeev, V.A. see

Vinogradova, S.A.

Topol, I.A. \& Polyakov, V.I. Method for molecular electronic state multiplet structure calculation in the space $\mathrm{Xa}$ SW-orbitals

Tran, C.T. see Bonomi, E.

2 (1991) 510

Trew, A.S., Wilding, N.S. \& Pawley, G.S. The phases of cyclohexane predicted by MD simulation

Trushin, O.S., Nayak, P., Stepanyuk, V.S. \& Katsnelson A.A. Molecular-dynamic computer simulation study of structural and transport properties of some amorphous alloys

2 (1991) 523

Tucker, J.W. see Drye, T.J. 2 (1991) 331

Turski, L.A. see Olko, M. 2 (1991) 425

Turtia, S.B. see Berdnicov, A.S. 2 (1991) 246 
V

Vicsek, T. see Bourbonnais, $R$. Vinogradova, S.A., Bernicov, A.S., Davydov, D.A., Golubok, A.O. \& Timofeev, V.A Experimental data computer treatment in scanning tunneling microscopy

Vogl, R.K. see Drevermann, $H$ von Boehm, J. \& Nieminen,

R.M. Nosé-Hoover molecular dynamics simulations of the pipe-diffusion mechanism in gold using many-atom forces

$\mathbf{w}$

Wesolowski, T. A discrete vs. continuum model of electronic polarization in proteins

Westermann, T. Optimization by numerical simulation of the focusing properties of selfmagnetically insulated ion diodes

Wielen, R. see Bien, $R$.

Wilcox, W. see Aoki, S

Wilding, N.S. see Trew, A.S.

Willers, I. Parallel computing:

Some activities in high energy physics

Wnek, M. \& Moscicki, K. Numerical study of the phase equilibria and molecular dynamics of stiff-chain polymers in solution
$2(1991) 272$

2 (1991) 719

2 (1991) 527

2 (1991) 328

2 (1991) 531

2 (1991) 536

2 (1991) 3

2 (1991) 829

2 (1991) 515

1 (1990) 343

2 (1991) 541
Wolf, M. see Edvardsson, $S$.

2 (1991) 348

$\mathbf{Y}$

Yudanin, B. \& Lax, M. Post adaptation for a numerical solution of the sphericallysymmetric Riemann problem

$1(1990) 285$

$\mathbf{Z}$

Zaitsevskii, A.V. \& Dement'ev, A.I. Molecular transition moment calculations using the intermediate operator quasidegenerate perturbation theory

Zaitsevskii, A.V. \& Dement'ev, A.I. Intermediate effective operators in valence-bond model spaces

Zelenskaya, N.S. see Belyaeva, T.L.

2 (1991) 546

Zelenskaya, N.S., Balashova, $O$ Yu \& Ovchinnikova, A.A. Program OLYMP for the analysis of direct nuclear reactions with semi heavy particles: The problem of automatic determination of integration range

2 (1991) 337 